\section{Irreversibility and the resurrection of the dead}

\author{
Ilya Prigogine and Yves Elskens
}

Une Incertaine Réalité: Le Monde Quantique, la Connaissance et la Durée. * By Bernard d'Espagnat. Gauthier-Villars, 17 rue Démy-Dumoncel, BP 50, 75661 Paris Cedex 14, France: 1985. Pp.310. Pbk FF98.

BERNARD d'Espagnat is well-known as the author of several excellent monographs on contemporary physics; his Conceptual Foundations of Quantum Mechanics (Addison-Wesley, 1976) and Nonseparability and the Tentative Descriptions of Reality (Phys. Rep. 110, 202-264; 1984) have become classics in their fields. Readers will therefore open his new book with anticipation, not least because its subtitle, Le Monde Quantique, la Connaissance et la Durée, promises an original discussion of such fundamental contemporary questions as causality and irreversibility.

d'Espagnat defends here the thesis that today's physics requires two irreducible notions of reality: on the one hand, an "independent" reality, and on the other an empirical reality. This is an idea which was also to be found in his preceding book, A la Recherche du Réel (GauthierVillars, 2nd Edn 1981). Using arguments originating in both epistemology and the natural sciences, d'Espagnat discusses this concept in detail but in terms that are accessible to the general reader. As would be expected, the "independent" reality is timeless while irreversibility appears only in empirical reality.

The thesis of two opposing realities is not new, nor is the response of most physicists to it. One is reminded of Léon Rosenfeld's "lay sermon", published in his Selected Papers (Reidel, 1976) in which he likened the attitude of many theoretical physicists to irreversibility to that of the Athenians towards Paul's preaching:

they quietly let him talk - until he came to the resurrection of the dead. Then they laughed at him and went away, saying: 'We'll listen to this another time'.

Similarly, physicists tend to find better things to do once irreversibility is mentioned: they know that irreversibility belongs only to the phenomenological world and (Rosenfeld again):

they assume strict reversibility in 'another world' - the world of atoms, of which until recently they had no better knowledge than the

*An Uncertain Reality: The Quantum World, Knowledge and Time. apostle could have had of paradise or the other place.

Be this as it may, the dualistic conception of reality does put physicists in a difficult position. Irreversible processes are seen to be of growing importance, not only on the phenomenological level, but also on that of fundamental descriptions (examples are the quantum theory of measurement, unstable elementary particles and cosmology in the early stage of the Universe). This trouble is aggravated when one notices that quantum processes cannot be described in terms of macroscopic space-time concepts when repeated measurements are involved (see, for example, B. Misra and E.C.G. Sudarshan's discussion of the quantum Zeno paradox which appeared in J. Math. Phys. 18, 756-763; 1977).

In the book, d'Espagnat makes frequent (and penetrating) reference to the work of our group in Brussels, but in his view this work establishes irreversibility only as an "instrumentalistic", phenomenological notion of reality. It is true that our understanding of the microscopic meaning of irreversibility, while dating back to Boltzmann, is still in an exploratory stage. However, in recent years a rigorous link has been developed between highly unstable classical dynamics (for example in Kolmogorov systems) and irreversibility. It turns out that the classical pictures of point dynamics, and of quantum theory, undergo basic modifications when the processes of observation and measurement are taken into account for unstable dynamical systems. This instability precludes the usual description in terms of trajectories and opens the way to a nonlocal, statistical description including irreversibility. The finite precision of observational data is not, in our opinion, specific to human measurement and must be included in every consistent theoretical scheme. This development tends to support the duality of reality suggested by d'Espagnat; curiously, he himself, at the end of the book, seems to lean towards acceptance of the notion of some deeper "réel vrai".

It is fascinating to see how today's physics echoes the great themes of Western philosophy; after all, d'Espagnat's two realities are close to the two "worlds", noumenal (intellectual) and phenomenal, introduced by Kant. More recently, of course, C.P. Snow examined the "two cultures". Anyone interested in what unites them at the deepest level will find d'Espagnat's book to be well worth reading.

Ilya Prigogine and Yves Elskens are in the Service de Chimie Physique II, Université Libre de Bruxelles, Campus Plaine CP 231, Boulevard du Triomphe, B 1050 Brussels, Belgium. Ilya Prigogine is also Ashbel Smith Regento Professor in the Department of Physics, University of Texas at Austin, Austin, Texas 78701, USA.

\section{Physics in concert}

\section{Pedro Waloschek}

QED: The Strange Theory of Light and Matter. By Richard P. Feynman. Princeton University Press:1986. Pp.158. \$18.50, $£ 13.40$.

THE message of this book is clear: Richard Feynman considers that the principles of quantum theory, and in particular of quantum electrodynamics (QED), should now be revealed to the world at large. Here he explains QED simply and without mathematics. Feynman, who earned the Nobel prize in 1965 for his contributions to QED, shows that this theory is as natural as numbers and that it describes 99 per cent of what happens with incredible accuracy.

In the past we used numbers to describe the world about us - and thereby made possible the myriad activities based on them - but now we use arrows. There is no magic about them and Feynman shows how easy they are to work with. By the end of the first chapter the reader is brought to understand that even the simplest phenomena, such as the reflection of light in a shop window, can only be described correctly using these little symbols. Newton's particle theory of light, or Maxwell's wave-theory (or both combined in some funny way), will never provide a correct prediction of, for example, the results of an experiment on reflection - if it is carried out with sufficient accuracy. The arrows, which move like the hands of a clock as time passes, are simple tools. They do not tell us why things happen, but how to describe them.

The book is made up of four chapters, which correspond to each of Feynman's Alix G. Mautner Memorial Lectures given at the University of California, and which are like four parts of a concert. First comes an adagio, then an andante. Next is the crescendo, followed by the overwhelming finale, which probably only those with prior knowledge will ever understand. But this is part of the game. First Feynman tells us how simple it all is. In Chapter 2 dawns the realization that more details must be taken into account. In Chapter 3 things become more complicated still, but the attentive reader remains convinced that everything, including the minutiae of atomic structure, can be calculated by using the arrow principle. Finally, Feynman tries to summarize our entire knowledge of particle physics in exactly 28 small-format pages - a valiant, hopeless enterprise, but resounding all the same.

The arrows are the probabilityamplitudes which have been used by physicists over the past few decades for all their computations. They correspond in mathe- 
matical language to complex numbers. But most physicists have never tried to find an intuitive way of thinking about them; we were always told that it could not be done. Feynman demonstrates that it can, and goes on to introduce another widely used tool in physics, his own Feynman graphs. In a simple and intuitive manner he shows how these diagrams can be "imagined", without understanding what is behind them but getting a good feeling for how they work. Years ago I heard a seminar by Feynman, in which he observed that many physicists used the graphs in much the same way that our ancestors would consult animal entrails for portents of the future. Now it seems that a new understanding has taken over; the graphs are taken as seriously as the arrows, and together they frame the world of present-day physics - the only valid description of nature we have.

Feynman's lectures must have been marvellous and they have been turned into an equally entrancing book, a vivid introduction to QED which is leavened and enlivened by his wit. Anyone with a curiosity about physics today should buy it, not only to get to grips with the deepest meaning of quantum theory but to possess a slice of history.

Pedro Waloschek is a senior scientist at DESY (Deutsches Elektronen Synchrotron), Notkestrasse 85, D-2000 Hamburg 52, FRG.

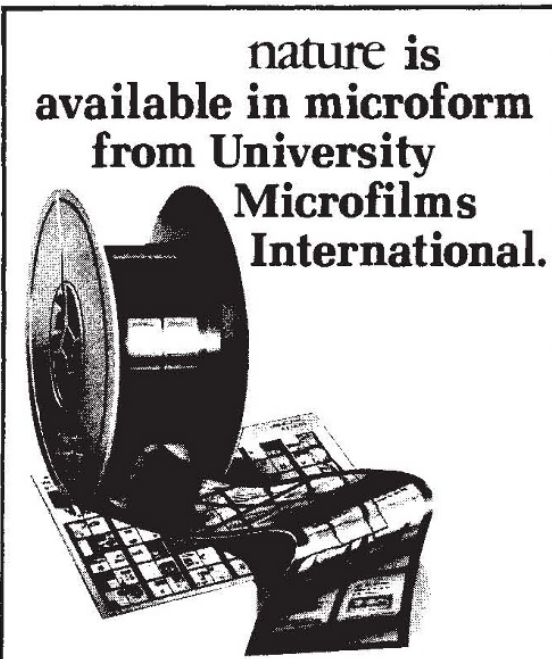

$\square$ Please send information about these titles:

Name

Company/Institution

Address

City

State

Zip

Phonel

Call toll-free 800-521-3044. In Michigan.

Alaska and Hawaii call collect 313-761-4700. Or mail inquiry to: University Microfilms International 300 North Zeeb Road, Ann Arbor, MI 48106.

\section{Schwinging through relativity}

\section{Abraham Pais}

Einstein's Legacy: The Unity of Space and Time. By Julian Schwinger. W.H. Freeman: 1986. Pp.250. £15.95.*

THE scientific community appreciates quite well the importance of conveying to a wider audience what science is about. So high are the demands of doing research, however, that few of those active in the field are willing to respond to this need. Doing science and writing about it are actually two distinct activities requiring distinct skills. Nevertheless I like to think that in principle the scientific craftsman is best qualified for popularization. All the many subtleties of any specific scientific subject cannot, in fact should not, be presented to those eager to obtain a first acquaintance with it. Awareness of these subtleties by the popular science writer will, however, temper his style, preventing him from couching his account in those overheated terms and exalted phrases so often used by those who do not really know what is going on.

Julian Schwinger is a pro, particularly well known for his seminal contributions to quantum electrodynamics, for which, with Feynman and Tomonaga, he was awarded the 1965 Nobel Prize in physics. Those as his other writings are marked by consummate mathematical skill. I must confess to considerable astonishment upon receiving his popular account Einstein's Legacy. That, I said to myself, is not the Schwinger I know well. I am happy to report, however, that his book makes for delightful and instructive reading. Technical demands on the reader do not exceed the most elementary algebra.

In the book, parts of Einstein's rich and varied legacy, on quantum theory, on Brownian motion, are touched on only in passing. As the subtitle The Unity of Space and Time indicates, the main thrust is here on relativity theory. The opening chapter "A Conflict Brought to Light" (several of the chapter titles are puns) serves as a prelude to the subject. It describes Michelson and Morley's failure to detect the speed with which the Earth drifts through the ether, that ubiquitous medium, for so long erroneously supposed to be necessary for explaining how light waves travel through space. It is explained why this negative result is of such positive value. Here and elsewhere the author acquaints the reader with the issues by first considering a more familiar analogy, in this case the problem of find-

*Published in the United States as a volume in the Scientific American Library. ing the speed of aircraft moving along with or perpendicular to the jet stream.

The next chapter, "Marking Time", introduces a variety of clocks: pendulums, vibrating atoms, beams circulating in cyclotrons. Soon we are in the midst of the results of relativistic measurements, so different from everyday expectations because of the relativity postulate that the speed of light is unaffected by the motion of the observer. Time intervals dilate, lengths contract - not really, but as seen by an observer at rest who sees, respectively, moving clocks and moving rods. Here, as elsewhere in the book, subtle concepts are elucidated by simple

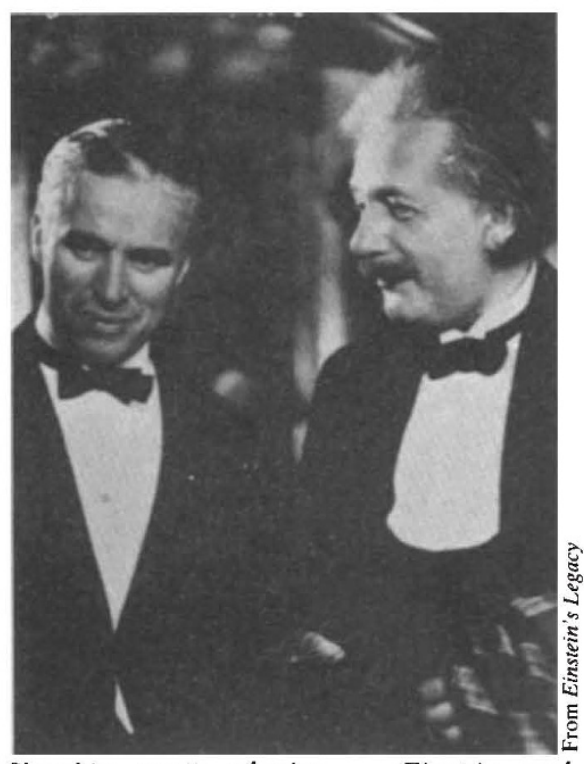

Showbiz meets physics - Einstein and Charles Chaplin in Hollywood, 1931

pictures. Thus the notion of causality is explained by describing the sequence of events at a race track. Cartesian coordinates are introduced by taking the reader on a walk through midtown Manhattan.

A chapter on $E=m c^{2}$ deals with the meaning of that relation in terms of conservation laws and with its applications to (in order of practical importance) chemical, atomic and nuclear reactions, including fission and fusion.

All this, so far, is special relativity which relates experiences of observers in uniform relative motion. As Einstein once said, that theory is child's play compared to general relativity which deals with nonuniform motion. It is particularly gratifying that the subject's intricacies, including glimpses of non-Euclidean and Riemannian geometries, and the theory's early successes, the bending and red shift of light, and the precession of the planet Mercury's perihelion are so well conveyed here in simple language. Altogether, this well printed and pleasingly illustrated book is an ideal gift for the curious non-expert. $\square$

Abraham Pais is a Professor in the Department of Physics, Rockefeller University, 1230 York Avenue, New York, New York 10021, USA. 